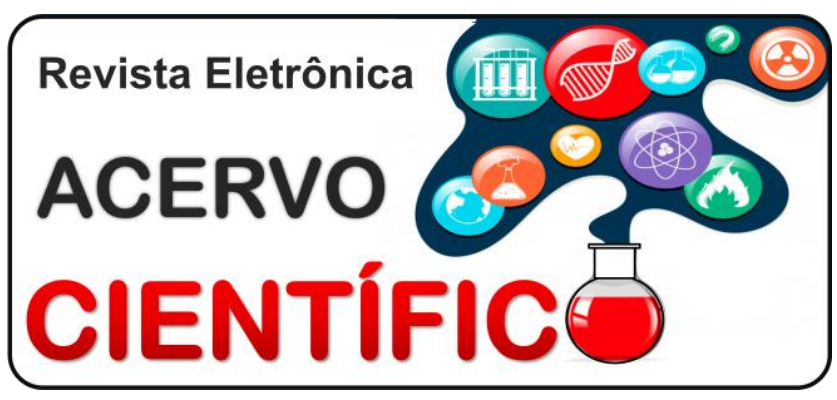

\section{REVISÃO BIBLIOGRÁFICA}

Recebido em: 8/2019

Aceito em: 9/2019

Publicado em: 10/2019

\title{
Aneurisma de artéria renal: uma revisão da literatura
}

\author{
Renal artery aneurysm: a literature review
}

Aneurisma de la arteria renal: una revisión de la literatura

lapunira Catarina Sant'Anna Aragão ${ }^{1 *}$, Felipe Matheus Sant'Anna Aragão1, André Luis Morelli Lustosa $^{1}$, Pedro Caldas Pereira ${ }^{1}$, Priscila Almada Torres ${ }^{1}$, Lívia Silva de Paula Faria ${ }^{1}$, José Aderval Aragão², Marcos Guimarães de Souza Cunha1.

Resumo: Esse artigo buscou discutir, através de uma revisão bibliográfica, a prevalência de aneurismas de artérias renais (AARs), seus fatores de risco, além dos melhores métodos diagnósticos, implicações clinicas, epidemiologia e tratamento cirúrgico, visando uma atualização de dados, principalmente, para os profissionais que atuam nesta área. 13 artigos foram selecionados e lidos na íntegra para compor os dados desta pesquisa. Os aneurismas de artéria renal são patologias bastante incomuns, e normalmente se manifestam de forma assintomática. Desse modo, o diagnóstico ocorre, principalmente, de maneira incidental, sendo a angiografia por ressonância magnética (Angio-RM) o método não-invasivo de eleição. Os estudos mais recentes preconizam o tratamento cirúrgico apenas na presença de sintomas manifestos, hipertensão não controlada ou risco presumido de ruptura. Caso contrário, o tratamento instituído deve ser conservador, sem intervenções cirúrgicas. Portanto, faz-se necessário o conhecimento sobre esta patologia, seu diagnóstico e tratamentos, em especial, para clínicos e cirurgiões que atuam nesta área.

Palavras-chave: Aneurisma; Artéria Renal; Diagnóstico; Terapêutica.

\begin{abstract}
This article aimed to discuss, through a literature review, the prevalence of renal artery aneurysms (RAAs), their risk factors, as well as the best diagnostic methods, clinical implications, epidemiology and surgical treatment, aiming at updating data, especially for professionals working in this area. 13 articles were selected and read in full to compose the data of this research. Renal artery aneurysms are very uncommon pathologies and usually manifest asymptomatically. Thus, the diagnosis occurs, mainly, incidentally, and magnetic resonance angiography is the noninvasive method of choice. The most recent studies recommend surgical treatment only in the presence of manifest symptoms, uncontrolled hypertension or presumed risk of rupture. Otherwise, the treatment instituted should be conservative, without surgical interventions. Therefore, knowledge about this pathology, its diagnosis and treatments are necessary, especially for clinicians and surgeons working in this area.
\end{abstract}

Keywords: Aneurysm; Renal Artery; Diagnosis; Therapeutics.

Resumen: Este artículo tuvo como objetivo discutir, a través de una revisión de la literatura, la prevalencia de aneurismas de la arteria renal (AARs), sus factores de riesgo, así como los mejores métodos de diagnóstico, implicaciones clínicas, epidemiología y tratamiento quirúrgico, con el objetivo de actualizar los datos, especialmente para los profesionales que trabajan en esta área. Se seleccionaron 13 artículos y se leyeron en su totalidad para componer los datos de esta investigación. Los aneurismas de la arteria renal son patologías muy poco frecuentes y generalmente se manifiestan asintomáticamente. Por lo tanto, el

${ }^{1}$ Centro Universitário de Volta Redonda (UNIFOA), Volta Redonda - RJ. *E-mail: icatarinasaragao@hotmail.com.

${ }^{2}$ Universidade Federal de Sergipe (UFS), Aracaju - SE. 
diagnóstico ocurre, principalmente, incidentalmente, y la angiografía por resonancia magnética es el método de elección no invasivo. Los estudios más recientes recomiendan el tratamiento quirúrgico solo en presencia de síntomas manifiestos, hipertensión no controlada o presunto riesgo de ruptura. De lo contrario, el tratamiento instituido debe ser conservador, sin intervenciones quirúrgicas. Por lo tanto, el conocimiento sobre esta patología, su diagnóstico y tratamientos es necesario, especialmente para los médicos y cirujanos que trabajan en esta área.

Palabras clave: Aneurisma; Arteria Renal; Diagnóstico; Terapéutica.

\section{INTRODUÇÃO}

Aneurismas de artérias renais (AARs) são patologias incomuns, ocorrendo em aproximadamente 0,09\% da população geral (THAM G, et al., 1982; HENKE PK, et al., 2001; NACIF MS, SANTOS AASMD, MARCHIORI E, 2006; GÓES JUNIOR AMO, et al., 2016). Sua história natural é pouco conhecida, mas é sabido que estão correlacionados a traumas, poliarterite nodosa (PAN), doença arteroesclerótica, infecções, displasias vasculares ou doença de Kawasaki; e têm maior prevalência em indivíduos do sexo feminino com mais de 45 anos de idade (THAM G, et al., 1982; HENKE PK, et al., 2001; BRITO LCM, et al., 2011; BARROS KJF, et al., 2014; MOLINARI GJDP, et al., 2014; ANTUNES I, et al., 2015).

Os AARs podem apresentar uma clínica de sintomas inespecíficos, mas, em sua maioria, são assintomáticos (THAM G, et al., 1982; BARROS KJF, et al., 2014; ANTUNES I, et al., 2015; GÓES JUNIOR AMO, et al., 2016). Dessa maneira, o diagnóstico é feito incidentalmente, através de exames de imagem solicitados para outras doenças, tais como: ressonância nuclear magnética, tomografia computadorizada e arteriografias (THAM G, et al., 1982; NACIF MS, SANTOS AASMD, MARCHIORI E, 2006; GÓES JUNIOR AMO, et al., 2016).

Estes aneurismas podem ocorrer em toda extensão da arterial renal, com prevalência nos ramos de primeira ordem e na sua bifurcação, podendo se apresentar com vários formatos, como fusiformes ou saculares, sendo estes os mais predominantes. Normalmente não apresentam calcificações, e a sua ruptura ou embolização são raros, com incidência de aproximadamente $5 \%$. Apesar de ainda não ser conhecida a real relação dos AARs com a hipertensão arterial sistêmica, esta é uma das comorbidades mais predominantes nas populações acometidas por estes aneurismas (THAM G, et al., 1982; HENKE PK, et al., 2001; BARROS KJF, et al., 2014).

Existem duas vertentes para o tratamento desses aneurismas, o tratamento conservador e o cirúrgico. As principais indicações para intervenções cirúrgicas são: Existem duas vertentes para o tratamento desses aneurismas, o tratamento conservador e o cirúrgico. O conservador está indicado em paciente assintomático, com aneurisma $<2 \mathrm{~cm}$ de diâmetro, já tratamento cirúrgico está indicado em pacientes sintomáticos, com hipertensão arterial sistêmica de difícil controle, quando seu diâmetro é $>2 \mathrm{~cm}$, na pan arterite nodosa (PAN) ou quando acomete mulheres em idade fértil, independentemente do tamanho do aneurisma, entretanto, o quesito do tamanho ainda é largamente debatido, e alguns autores consideram outros valores como critério seletor 1,5,7,8 (HENKE PK, et al., 2001; BRITO LCM, et al., 2011; BARROS KJF, et al., 2014; MOLINARI GJDP, et al., 2014).

Atualmente, para que haja uma correção endovascular satisfatória aliado a um acompanhamento pósoperatório eficiente, é de fundamental importância que se tenha um minucioso planejamento pré-operatório para a escolha dos materiais e determinação da abordagem cirúrgica, através dos atuais métodos de diagnóstico e rastreio. Este conhecimento sobre o aneurisma facilita a escolha de um tratamento eletivo e que apresente menor morbimortalidade. Logo, o diagnóstico incidental e os protocolos de rastreamento contribuem significativamente para a diminuição da mortalidade e complicações associadas aos AARs (NACIF MS, SANTOS AASMD, MARCHIORI E, 2006; BARROS KJF, et al., 2014; GÓES JUNIOR AMO, et al., 2016).

Este estudo teve como objetivo identificar a prevalência de AARs, seus fatores de risco, além dos melhores métodos diagnósticos, implicações clinicas, epidemiologia e tratamento cirúrgico, visando uma 
atualização de dados, principalmente, para os profissionais que atuam nesta área. Foi realizado um levantamento bibliográfico nas bases de dados eletrônicos SciELO e PubMed, com os descritores "aneurisma", "artéria renal", "diagnóstico", "terapêutica" e seus correspondentes termos em língua inglesa. 13 artigos foram selecionados e lidos na íntegra para compor os dados desta pesquisa.

\section{REVISÃO BIBLIOGRÁFICA}

\section{Epidemiologia}

Os AARs são achados raros e têm uma incidência de aproximadamente 0,09\% 1-5. Esta patologia tem uma maior predominância nas mulheres, superando quase que o dobro do valor encontrado nos homens, isto se dá devido a sua forte associação com a fibrodisplasia muscular renal (HENKE PK, et al., 2001; BARROS KJF, et al., 2014). Contudo, o estudo de Góes Junior AMO, et al. (2016), realizado numa população com mais de 50 anos, encontrou uma incidência de $0,08 \%$, está se concentrando no sexo masculino.

A maioria dos pacientes é diagnosticada incidentalmente, em virtude de se tratar de um quadro clínico predominantemente assintomático. Estes aneurismas costumam ser solitários, sem preferência estabelecida por um dos dimídios e seu tamanho e localização podem variar de indivíduo para indivíduo (THAM G, et al., 1982; HENKE PK, et al., 2001; BARROS KJF, et al., 2014; ANTUNES I, et al., 2015; GÓES JUNIOR AMO, et al., 2016).

Existem alguns fatores de risco e doenças vasculares associadas, dentre eles estão: fibrodisplasia da artéria renal, lesões oclusivas arterioscleróticas concomitantes (ex.: doença vascular periférica, doença arterial coronariana), história ou uso atual de tabaco, e a presença de outros aneurismas, incluindo os da aorta e da artéria esplênica. Além disso, a hipertensão arterial parece ser um fator habitual nesses pacientes, todavia ainda não se sabe quais os mecanismos que medeiam essa correlação (THAM G, et al., 1982; HENKE PK, et al., 2001).

$\mathrm{Na}$ pesquisa de Henke PK, et al. (2001) foram encontrados diferentes caracteres histológicos, como alterações displásicas, alterações fibrosas não específicas e alterações ateroscleróticas acentuadas, sendo esta última a mais prevalente. Ademais, relatos anteriores sugeriram que a arteriosclerose nesses aneurismas é um fenômeno secundário e não primário (THAM G, et al., 1982; HENKE PK, et al., 2001).

É importante também salientar a existência dos falsos aneurismas (FA) da artéria renal, esses são normalmente associados a traumatismo prévio (NACIF MS, SANTOS AASMD, MARCHIORI E, 2006; ANTUNES I, et al., 2015).

$\mathrm{Na}$ análise de Antunes I, et al. (2015), houve 100\% de associação entre os casos de FA e história recente de algum procedimento urológico, como: nefrectomia parcial, nefrolitotomia percutânea ou biopsia renal.

\section{Diagnóstico}

O diagnóstico de AARs usualmente acontece de forma incidental por meio de exames de imagem, como ressonância magnética, tomografia computadorizada e estudos arteriográficos. No estudo de Nacif MS, Santos AASMD e Marchiori E (2006), a principal indicação que levou a realização do exame foi a hipertensão arterial sistêmica (26,7\%), seguida de dor abdominal (12,5\%), aneurisma da aorta abdominal $(10,7 \%)$ e estenose da artéria renal (8,9\%). Para Henke PK, et al. (2001), este valor foi de $42 \%$ de arteriografias realizadas por suspeita de hipertensão renovascular.

A tomografia computadorizada (TC), possibilita a avaliação das características dos aneurismas e da própria anatomia vascular renal, sendo um exame importante no planejamento e acompanhamento pré e pós-operatório. Entretanto, a arteriografia pré-operatória deve ser realizada nos casos em que não for possível constatar todas as informações necessárias para um manuseio endovascular correto e eficiente. Ademais, em situações de aneurismas complexos, a angiografia rotacional, com reconstrução 
tridimensional, torna-se essencial na avaliação da angioarquitetura vascular renal, mais complexos e que necessitem de uma melhor avaliação imagenológica (NACIF MS, SANTOS AASMD, MARCHIORI E, 2006; BUSATO CR, et al., 2008; BARROS KJF, et al., 2014).

A TC é um recurso que tem a sua eficácia limitada no pós-operatório, devido a sua visualização inadequada das molas e dos vazamentos, em virtude dos artefatos provocados pela presença de metal. Nesses casos, a ultrassonografia Doppler (USG-D) e a angioressonância são os métodos mais indicados para o seguimento desses indivíduos (THAM G, et al., 1982; NACIF MS, SANTOS AASMD, MARCHIORI E, 2006; BARROS KJF, et al., 2014). A angiografia por ressonância magnética (angio-RM) é um método que tem se mostrado seguro e eficaz na avaliação das artérias renais, com as vantagens de não se usar meio de contraste iodado e potencialmente alérgeno, não usar radiação ionizante ou os riscos de uma cateterização (NACIF MS, SANTOS AASMD, MARCHIORI E, 2006; GÓES JUNIOR AMO, et al., 2016).

\section{Tratamento}

A decisão de intervir cirurgicamente em um AAR deve ser baseada em vários fatores, incluindo a idade do paciente, sexo, gravidez antecipada em pacientes do sexo feminino, e as características anatômicas do AAR, incluindo seu tamanho. Este último aspecto tem sido exaustivamente debatido nos últimos anos, e as opiniões dos autores variam entre valores de 1.3 a $2.0 \mathrm{~cm}$. Por conseguinte, destacam-se como os principais fatores para indicação cirúrgica a presença de sintomas manifestos, hipertensão não controlada ou risco presumido de ruptura (THAM G, et al., 1982; RUNDBACK JH, et al., 2000; HENKE PK, et al., 2001; WAJNBERG E, AQUINO D, SPILBERG G, 2010; BRITO LCM, et al., 2011; BARROS KJF, et al., 2014).

Todos os casos de AARs devem ser tratados na presença de determinados critérios, tais como: pacientes sintomáticos; AAR maior que $2 \mathrm{~cm}$ ou crescimento aneurismático documentado; AAR em mulheres em idade fértil ou em gestação; AAR com embolização distal documentada; AAR associado à estenose significativa e à má perfusão renal. Do contrário, esses pacientes devem ser observados de forma clínica e conservadora, sem intervenções cirúrgicas (THAM G, et al., 1982; HENKE PK, et al., 2001; WAJNBERG E, AQUINO D, SPILBERG G, 2010; BARROS KJF, et al., 2014).

Atualmente, os AARs têm obtido resultados muito satisfatórios, com uma menor morbimortalidade e preservação da anatomia vascular, isso se deve a constante evolução das opções de tratamento e o aperfeiçoamento de novas técnicas. (THAM G, et al., 1982; RUNDBACK JH, et al., 2000; HENKE PK, et al., 2001; NOGUEIRA C, et al., 2012; BARROS KJF, et al., 2014).

Existem diversos procedimentos que podem ser realizados, dentre eles aneurismectomia e fechamento angioplástico da artéria renal ou reimplante de artéria renal segmentar, aneurismectomia e reconstrução da artéria renal com enxerto, nefrectomia planejada, uso de endopróteses, stents, molas, etc (RUNDBACK JH, et al., 2000; HENKE PK, et al., 2001; BUSATO CR, et al., 2011; BARROS KJF, et al., 2014; GENZINI T, et al., 2014).

Rundback JH, et al. (2000) classificou os AARs em 3 tipos de acordo com a sua localização para sistematizar a escolha dos procedimentos corretos para cada tipo de aneurisma. São eles: tipo I (aneurismas localizados na artéria renal principal), tipo II (aneurismas localizados no hilo) e tipo III (aneurismas intrarrenais).

Para os AARs do tipo I, existem as opções de tratamento por endoprótese ou embolização seletiva com mola, esta última podendo ser utilizada nos três tipos descritos. Para os AARs do tipo II, as escolhas variam entre stents ou molas e endopróteses. Já para os AARs do tipo III, sendo estes os que possuem maior risco de ruptura, a preferência se restringe à embolização superseletiva de ramo segmentar renal com uso de $\mathrm{N}$ butil cianoacrilato (RUNDBACK JH, et al., 2000; BARROS KJF, et al., 2014).

\section{Prognóstico}

Os pacientes que são selecionados para uma abordagem conservadora costumam ser assintomáticos, com aneurismas de pequeno tamanho e sem complicações. Esse grupo costuma manter-se estável, sem grandes prejuízos, e são raros os casos de ruptura (THAM G, et al., 1982; HENKE PK, et al., 2001). No 
estudo de Henke PK, et al. (2001), após um seguimento médio de 72 meses desses indivíduos, nenhuma ruptura do AARs foi documentada.

Já para o grupo cirúrgico, um benefício observado é o melhor controle da hipertensão arterial, porém este é um critério de difícil quantificação. No entanto, esse efeito deve ser considerado potencialmente importante, visto que as sequelas hipertensivas são insidiosas, e as complicações muitas vezes permanentes. Para todos os pacientes submetidos a alguma abordagem cirúrgica parece haver algum grau de redução da pressão arterial, no entanto certos procedimentos aparentam possuir um menor efeito redutor, como a nefrectomia, quando comparada a reconstruções arteriais. O modo de reparo, seja aneurismectomia com fechamento angioplásico ou bypass, não pareceu relevante em termos de hipertensão: ambas as intervenções terapêuticas resultaram em reduções significativas da pressão arterial. Em alguns estudos, houveram até relatos de redução no número de medicamentos anti-hipertensivos ou em suas doses (THAM G, et al., 1982; HENKE PK, et al., 2001; BARROS KJF, et al., 2014).

Dentre as principais complicações perioperatórias estão a hemorragia pós-operatória e a trombose venosa profunda, entretanto existem descrições de pneumonia, bloqueio cardíaco de terceiro grau e pancreatite pós-operatória. No período pós-operatório podem existir anormalidades técnicas posteriores que necessitem de intervenção adicional, como angioplastia anastomótica percutânea, reoperação precoce para trombose do enxerto e embolização percutânea por ramo segmentar estenótico, contudo, essas complicações não são freqüentes (THAM G, et al., 1982; HENKE PK, et al., 2001; BRITO LCM, et al., 2011; NOGUEIRA C, et al., 2012).

$\mathrm{Na}$ análise de Henke PK, et al. (2000) não foram observadas diferenças significativas em termos de permeabilidade a longo prazo ou necessidade de intervenções secundárias entre os pacientes que tiveram aneurismectomia e bypass em comparação com aqueles que tiveram aneurismectomia e fechamento angioplástico primário da artéria renal.

\section{CONSIDERAÇÕES FINAIS}

Os aneurismas de artéria renal são bastante incomuns e podem estar associados a doenças vasculares e fatores de risco, representando uma patologia que se manifesta, em sua grande maioria das vezes, de forma assintomática. Desse modo, a literatura mostra que o diagnóstico ocorre, usualmente, de maneira incidental por meio de exames de imagem solicitados para outras doenças ou indicados na presença de sintomas como hipertensão arterial, sendo a Angio-RM um método que tem se mostrado seguro e eficaz na avaliação das artérias renais. Os estudos mais recentes preconizam o tratamento cirúrgico apenas na presença de certos fatores, tendo como os principais, a presença de sintomas manifestos, hipertensão não controlada ou risco presumido de ruptura. Caso contrário, o tratamento instituído deve ser conservador, sem intervenções cirúrgicas. Portanto, faz-se necessário o conhecimento sobre esta patologia, seu diagnóstico e tratamentos, em especial, para clínicos e cirurgiões que atuam nesta área.

\section{REFERÊNCIAS}

1. ANTUNES I, et al. Falsos aneurismas traumáticos da artéria renal - a nossa experiência. Angiologia e Cirurgia Vascular, 2015; 11(4):199-203.

2. BARROS KJF, et al. Técnicas e táticas no tratamento endovascular do aneurisma da artéria renal. Revista Brasileira de Cardiologia Invasiva, 2014; 22(1): 64-72.

3. BRITO LCM, et al. Tratamento de aneurisma da artéria renal por embolização e técnica de remodelamento de colo: relato de caso. Jornal Vascular Brasileiro, 2011; 10(2): 181-184.

4. BUSATO CR, et al. Aneurisma de artéria renal em rim transplantado: reparo ex vivo e reimplante do enxerto. Jornal Vascular Brasileiro, 2008; 8(1): 89-91.

5. GENZINI T, et al. Renal auto transplantation to treat renal artery aneurysm: case report. São Paulo Medical Journal, 2014; 132(5): 307-310.

6. GÓES JUNIOR AMO, et al. Achados incidentais de aneurismas torácicos e abdominais. Jornal Vascular Brasileiro, 2016; 15(2): 106-112. 
7. HENKE PK, et al. Renal artery aneurysms: a 35-year clinical experience with 252 aneurysms in 168 patients. Annals of Surgery, 2001; 234(4): 454-463.

8. MOLINARI GJDP, et al. Proposta de correção virtual geométrica da projeção ostial da artéria renal no estudo operatório de aneurismas infra-renais: resultados iniciais de um estudo piloto. Revista Brasileira de Cirurgia Cardiovascular, 2014; 29(1): 78-82.

9. NACIF MS, SANTOS AASMD, MARCHIORI E. Angiografia por ressonância magnética na avaliação das artérias renais: achados de imagem. Radiologia Brasileira, 2006; 39(4): 523-258.

10. NOGUEIRA C, et al. Aneurisma da Artéria Renal: cirurgia renal ex-vivo - a propósito de um caso clínico. Angiologia e Cirurgia Vascular, 2012; 8(3): 146-150.

11. RUNDBACK JH, et al. Percutaneous stent-graft management of renal artery aneurysms. Journal of Vascular and Interventional Radiology, 2000; 11(9): 1189-93.

12. THAM G, et al. Renal artery aneurysms: natural history and prognosis. Annals of Surgery, 1982; 197(3): 348-352.

13. WAJNBERG E, AQUINO D, SPILBERG G. Experiência preliminar com o uso da técnica de "remodelagem de colo" para tratamento endovascular de aneurismas complexos da artéria renal. Radiologia Brasileira, 2010; 43(1): 29-33. 